\title{
Use of the bioactive resorbable plate system for zygoma and zygomatic arch replacement and fixation with modified Crockett's method for maxillectomy: A technical note
}

\author{
SHINTARO SUKEGAWA ${ }^{1}$, TAKAHIRO KANNO ${ }^{1,2}$, AKANE SHIBATA $^{1}$, KENICHI MATSUMOTO ${ }^{1}$, \\ YUKA SUKEGAWA-TAKAHASHI $^{1}$, KYOUSUKE SAKAIDA ${ }^{1}$ and YOSHIHIKO FURUKI ${ }^{1}$ \\ ${ }^{1}$ Division of Oral and Maxillofacial Surgery, Kagawa Prefectural Central Hospital, Takamatsu, Kagawa 760-8557; \\ ${ }^{2}$ Department of Oral and Maxillofacial Surgery, Shimane University Faculty of Medicine, Shimane 693-8501, Japan
}

Received July 14, 2016; Accepted April 24, 2017

DOI: $10.3892 / \mathrm{mco} .2017 .1269$

\begin{abstract}
As a surgical approach targeting the pterygopalatine fossa following maxillary cancer due to tumor invasion, Crockett's method is conventional and useful. However, if the tumor is confined to the area between the maxilla and pterygopalatine fossa, it is not necessary to include the zygomatico-orbital in the access osteotomy, and the orbital floor may be preserved. Depending on the range of tumor invasion, the current study reports a more minimally invasive, modified Crockett's surgery that may be considered, which includes resection with modified osteotomy lines and repositioning with fixation of the zygoma and zygomatic arch following maxillary cancer ablation. In addition, the majority of patients with advanced maxillary cancer may require postoperative radiotherapy or chemoradiotherapy following maxillectomy according to several guidelines. Therefore, using a low-profile bioactive resorbable plate system as a method of repositioning and fixing the resected and preserved zygoma and zygomatic arch may be more effective in this modified Crockett's method for maxillectomy.
\end{abstract}

\section{Introduction}

As a surgical approach targeting the pterygopalatine fossa following maxillary cancer due to tumor invasion, Crockett's method is conventional and useful (1). In Crockett's method, the zygomatico-orbital bones are cut through the inferior orbital fissure from the zygomaticotemporal sutures, after which the separated bone contains zygoma and orbit, followed by repositioning and fixing with metal plates and screws. Therefore, if it

Correspondence to: Dr Shintaro Sukegawa, Division of Oral and Maxillofacial Surgery, Kagawa Prefectural Central Hospital, 1-2-1 Asahi-cho, Takamatsu, Kagawa 760-8557, Japan

E-mail: gouwan19@gmail.com

Key words: bioactive resorbable plate, Crockett's method, maxillary cancer, zygoma, zygomatic arch is necessary to simultaneously cut the maxilla and include the orbital floor, the Crockett's method may be effective. However, if the tumor is confined to the area between the maxilla and pterygopalatine fossa, it may not be necessary to include the zygomatico-orbital in the osteotomy and the orbital floor may be preserved accordingly. Depending on the range of maxillary tumor invasion, a more minimally invasive modified Crockett's surgery, which includes resection of the zygoma and zygomatic arch, may be applicable.

Furthermore, the majority patients with advanced oral, head and neck cancer are likely to require postoperative radiotherapy or chemoradiotherapy following maxillectomy according to the several clinical guidelines, such as the NCCN Clinical Practice Guidelines in Oncology (NCCN Guidelines) (2); therefore, it is probable that the use of a conventional titanium metal plate may interfere with and further complicate radiation field problems, such as scattered radiation in the maxilla, including the periorbital regions (3). There remains a requirement for modification of methods to compensate for these disadvantages. Therefore, the resorbable or bioactive resorbable plate application may be very effective for these patients. However, despite the notable advances in conventional resorbable osteosynthesis systems, there have been no significant improvements in their strength compared with that of titanium plate systems; sufficient thickness is still required to maintain strength, and complications, such as palpability, are associated with the thickness of resorbable plate systems in midfacial regions $(4,5)$.

As a recent clinical study reveals the feasible applicability of a newly developed low-profile bioactive resorbable plate system for periorbital regions (6), the current study applied such bioactive resorbable plate systems to zygoma and zygomatic arch replacement and fixation in this modified Crockett's method, including modified osteotomy line setting for both the zygoma and zygomatic arch considering oromandibular functional loads, for maxillectomy surgery to treat patients with advanced maxillary squamous cell carcinoma.

\section{Patients and methods}

The surgical procedure was performed as follows. The skin incisions are similar to those used in the Weber-Fergusson 
procedure, ascending through the upper lip, skirting the ala of the nose, following the nasofacial groove to the inner canthus of the eye and traversing the lower orbital margin to the outer canthus (Fig. 1). The mucosa of the upper buccal sulcus was incised down to the bone, and the skin flap was dissected back as far as the malar buttress. The infra-orbital nerve was divided just clear of the bone, which is the only irreversible step in this approach. The periosteum was then stripped, the infraorbital neurovascular bundle was disconnected and the facial flap was reflected. In addition to stripping the periosteum of the zygoma and zygomatic arch, the articular eminence was exposed. In the oral cavity, the incision line was located $\geq 10 \mathrm{~mm}$ from the cancerous tissue, with the incision leading to the bone surface.

The rear portion of the zygomatic arch was cut from the front of the articular tubercle diagonally upward using a saw. In addition, as the forward area of the zygomatico-maxilla was cut in an L-shape and this rear area was cut upwards diagonally. This was performed as it is easy to reposition to allow the oromandibular to be functionally stable in the mouth opening and provide good occlusion (Fig. 2). Then, the zygoma was disconnected from the front of the masseter muscle attachment in an L-shape (Fig. 3). The resected bone pieces were reflected downward along with the masseter muscle as a pedicle, so that the temporal muscle on the inside was exposed. After separating the periosteum at the front edge of the mandible, and exposing the bone surface, the coronoid process was cut in a reverse L-shape using a saw. The resected coronoid process was included for cancer ablation, or expanded upward with the temporal muscle according to the lateral cancer invasion. The maxillary artery was followed on the central side and ligated. Then, the forward, upward, and palatal sides of the maxilla were excised using a bone saw. The lateral and medial plates of the sphenoid are excised using the saw and chisels. The excised maxilla, including the tumor, the lateral pterygoid and medial pterygoid muscle were disconnected and the excised tissue was removed under clear surgical views with this feasible Crockett's method. The excised zygoma and zygomatic arch were then repositioned and fixed using low-profile bioactive resorbable plates and screws (Super FIXSORB MX ${ }^{\circledR}$; Takiron Co., Ltd., Osaka, Japan) (Fig. 4). Finally, the cheek flap was closed in layers.

\section{Discussion}

For maxillary resection, a transoral approach is also possible. However, it is difficult to control the bleeding in cases where the resection range extends to the pterygopalatine fossa. Therefore, an extra-oral approach is necessary to create a clearly visible operative field (7). Crockett's method, which results in clear surgical visualization of the pterygopalatine fossa by deploying the masseter downward following bone resection, is very useful and safe for maxillectomy operation. However, if the cancer is confined to the area between the maxilla and pterygopalatine fossa, it is not necessary to include the zygomatico-orbitals in the osteotomy, and the orbital floor may be preserved. The more minimally invasive modified Crockett's method, which may include the resection of the zygoma and zygomatic arch, also effectively achieves a sufficiently clear view of the surgical field in maxillectomy cancer ablation.

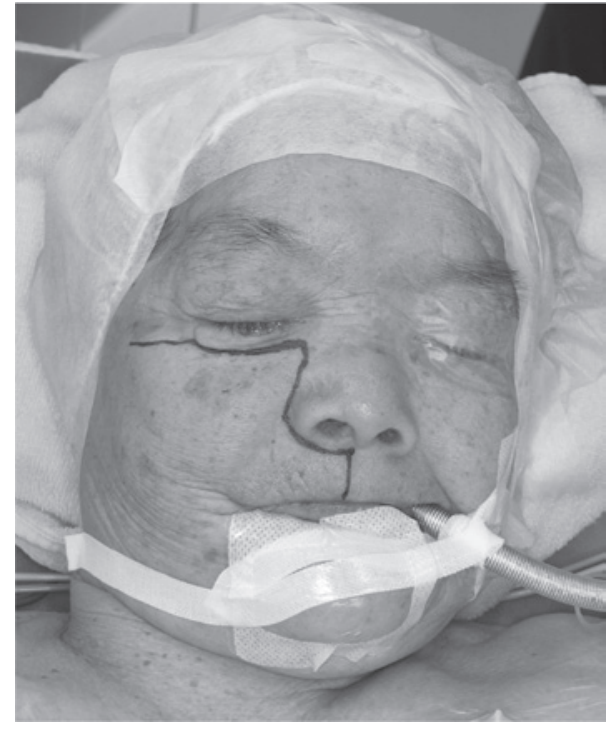

Figure 1. Setting the incision line. The incision is extended to the lateral sides in conformity with the Weber-Fergusson incision method.

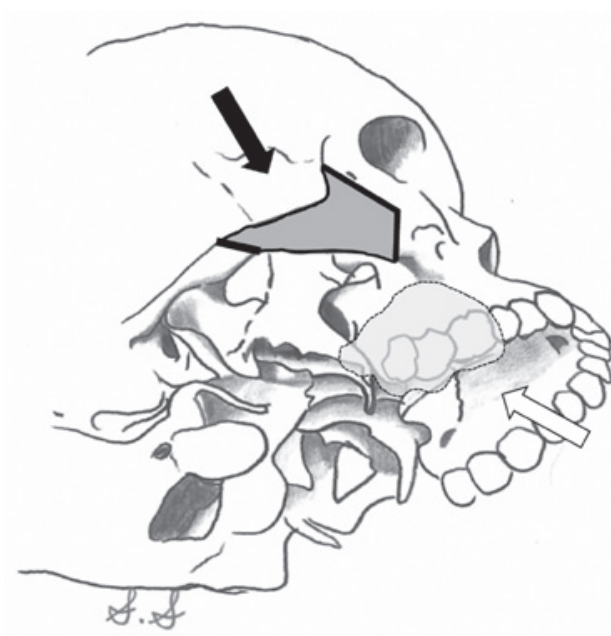

Figure 2. The modified osteotomy lines as the forward area of the zygomatico-maxilla is cut in an L-shape, and the rear area is cut upwards diagonally (black arrow). The white arrow shows the maxillary cancer invasion to the pterygopalatine fossa.

The majority of patients with advanced maxillary cancer undergo tumor ablative surgery first, followed by adjuvant therapy postoperatively using radiotherapy or chemoradiotherapy based on the maxillectomy specimen pathological examination according to the clinical guidelines in oral, head and neck cancers $(2,8)$, which may be the most advanced maxillary cancer types. However, metal artifacts are mostly due to quantum noise, scattered radiation and beam hardening (9). In radiation oncology, target delineation and tissue electron density as indicated by computed tomography (CT) Hounsfield numbers have a critical role in assuring CT-based treatment planning accuracy. CT images of patients with metal implants typically suffer from artifacts in the form of high electron density, low electron density, cupping and capping (10). Therefore, for more accurate and less complicated postoperative irradiation field setting, particularly in the maxilla of the midface close to the orbital contents and 

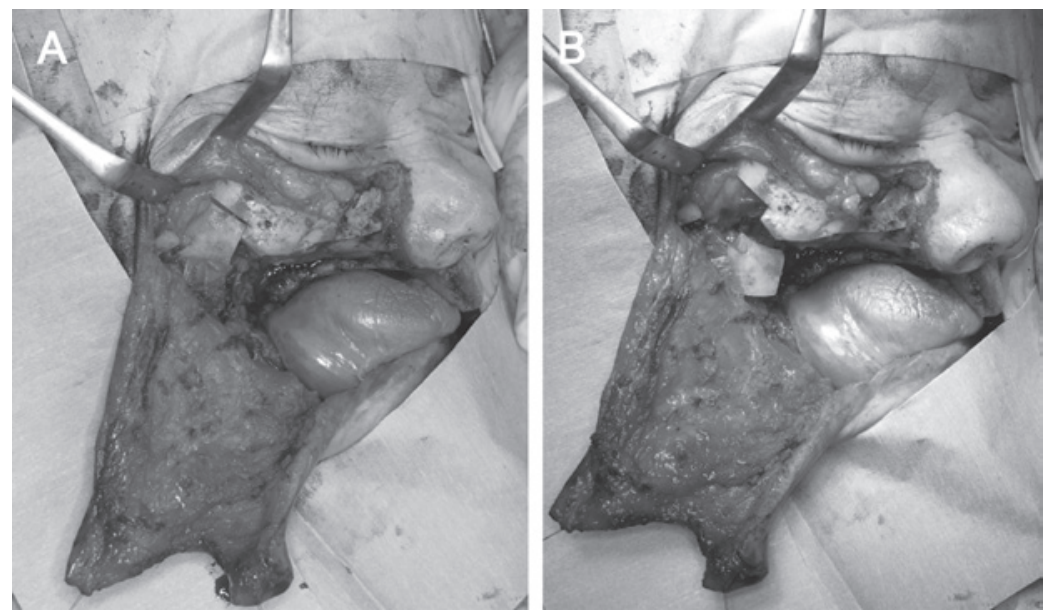

Figure 3. (A) The rear portion of the zygomatic arch is cut from the front of the articular tubercle diagonally upward using a saw. (B) Then, the zygoma is disconnected from the front of the masseter muscle attachment portion in an L-shape.

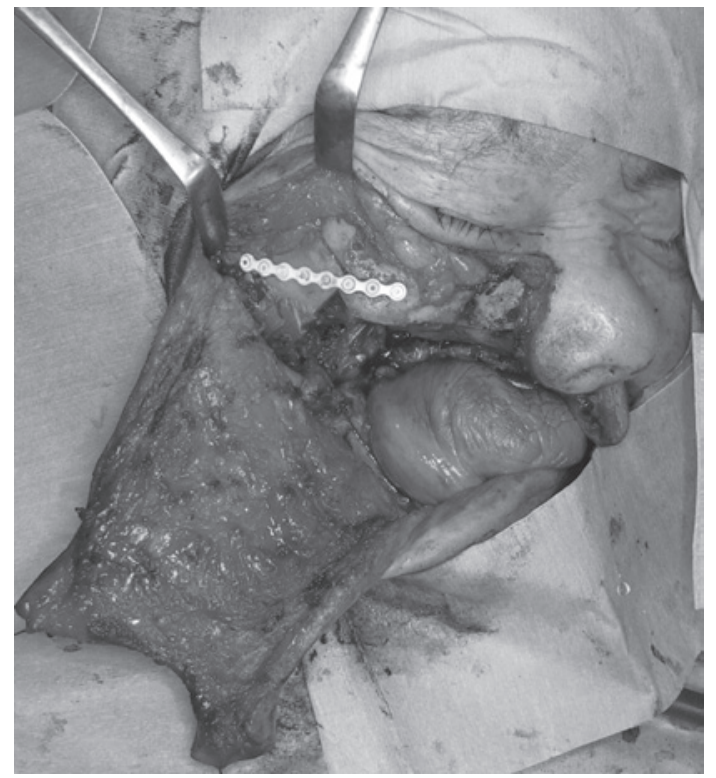

Figure 4. Excised zygoma and zygomatic arch are repositioned, and fixed using a low-profile bioactive bioresorbale plate (Super FIXSORB MX ${ }^{\circledR}$ ).
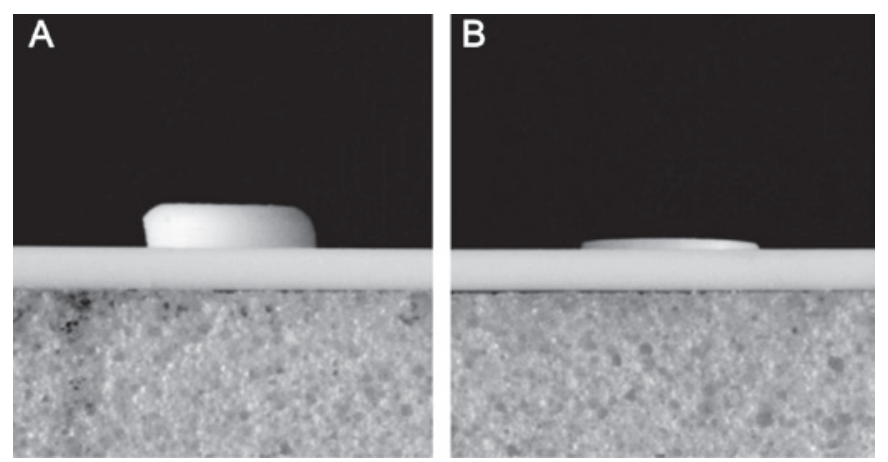

Figure 5. (A) Conventional screw and (B) improved screw. As the screw head is small, a low-profile plate system has been achieved.

facial skin, bioresorbable plates may be considered to be more clinically useful than conventional titanium metal plates. In this respect, to compensate for the biomechanical strength in oromandibular function, the current study introduced modified osteotomy to Crockett's maxillectomy method, where the forward area of the zygomatico-maxilla is osteotomized in an L-shape and the rear area is cut upwards diagonally, which may aid in repositioning with fixation for a functionally stable oromandibular, even with functional loads in mouth opening, and allow good occlusion.

The bioactive resorbable plate system was subsequently selected (Super FIXSORB MX ${ }^{\circledR}$ ) to reposition and fix the resected zygoma and zygomatic arch following maxillary cancer ablation. This new product is a composite of fine particles of unsintered hydroxyapatite (u-HA) and poly-L-lactide (PLLA). The properties of this material have the following clinical advantages, as has been recently reported, in feasible applicability in maxillofacial trauma surgery (11) and in particular in the midface for periorbital fracture fixation for reconstruction (6) with good clinical results even in long-term follow-ups (12): Its mechanical strength closely approximates that of cortical bone, it has osteoconductive properties and it makes direct contact with the bone. Furthermore, due to its bioactive and biodegradable properties, the u-HA/PLLA composite has the potential for total replacement by bone (13). Furthermore, plate stability has been demonstrated for intensive irradiation-like postoperative radiation therapy (14). The conventional resorbable plate is composed of PLLA alone. Following intensive irradiation, pure PLLA loses its mechanical strength and contains cracks throughout. Decreasing mechanical properties are considered to be correlated with the degradation of PLLA under radiation (15). However, excess palpability has been reported as a complication of surgery, as this material is thicker than conventional plate systems (11). Therefore, this improved plate system is low profile, as the step of the plate and the screw is small, and it has a flat structure (Fig. 5). In addition, the new plate system reported in the present technical note does not require an area for gripping a conventional screw head. Thus, as the area of the screw head is smaller, it is possible to create a thinner product.

Due to the thin skin over the zygomatic arch, palpability of the plate system is a strong possibility. Therefore, this bioactive resorbable plate system is considered to be very effective in this surgical procedure. 
In conclusion, a minimally invasive modified Crockett's method for maxillary cancer ablation located between the maxilla and pterygopalatine fossa, in which the zygoma and zygomatic arch are resected, is a useful technique. Additionally, a newly developed low-profile bioactive resorbable plate system for repositioning and fixing the resected zygoma and zygomatic arch with modified osteotomy lines may be effective in this maxillary cancer treatment.

\section{References}

1. Crockett DJ: Surgical approach to the back of the maxilla. Br J Surg 50: 819-821, 1963.

2. National Comprehensive Cancer Network: NCCN Clinical Practice Guidelines in Oncology. Head and Neck Cancers. Version 2.2013, 2013. https://www.nccn.org/professionals/physician gls/f guidelines.asp. Accessed August 22, 2013.

3. Thaller SR, Lee T and Tesluk H: Polyglyconate fixation successfully stabilizes zygomatic osteotomies in a nonhuman primate. J Craniofac Surg 6: 459-464, 1995.

4. Menon S and Chowdhury S: Evaluation of bioresorbable vis-à-vis titanium plates and screws for craniofacial fractures and osteotomies. Med J Armed Forces India 63: 331-333, 2007.

5. Buijs GJ, van Bakelen NB, Jansma J, de Visscher JG, Hoppenreijs TJ, Bergsma JE, Stegenga B and Bos RR. A randomized clinical trial of biodegradable and titanium fixation systems in maxillofacial surgery. J Dent Res 91: 299-304, 2012.

6. Kanno T, Tatsumi H, Karino M, Yoshino A, Koike T, Ide T and Sekine J: Applicability of an unsintered hydroxyapatite particles/poly-L-lactide composite sheet with tack fixation for orbital fracture reconstruction. J Hard Tissue Biol. 25: 329-334, 2016.

7. Pogrel MA: The management of salivary gland tumors of the palate. J Oral Maxillofac Surg 52: 454-459, 1994.
8. Pfister DG, Spencer S, Brizel DM, Burtness B, Busse PM, Caudell JJ, Cmelak AJ, Colevas AD, Dunphy F, Eisele DW, et al: Head and neck cancers, version 1.2015. J Natl Compr Canc Netw 13: 847-855; quiz 856, 2015.

9. Hsieh J: Image artifacts, causes and correction. In: Medical CT and Ultrasound, Current Technology and Application. Goldman LW and Fowlkers JB (eds). Madison: Advanced Medical Publishing, pp487-518, 1995.

10. Kim Y, Tomé WA, Ba M, McNutt TR and Spies L: The impact of dental metal artifacts on head and neck IMRT dose distributions. Radiother Oncol 79: 198-202, 2006.

11. Sukegawa S, Kanno T, Katase N, Shibata A, Takahashi Y and Furuki Y: Clinical evaluation of an unsintered hydroxyapatite/poly-L-lactide osteoconductive composite device for the internal fixation of maxillofacial fractures. J Craniofac Surg 27: 1391-1397, 2016.

12. Sukegawa S, Kanno T, Kawai H, Shibata A, Takahashi Y, Nagatsuka $\mathrm{H}$ and Furuki Y: Long-term bioresorption of bone fixation devices made from composites of unsintered hydroxyapatite particles and poly-L-Lactide. J Hard Tissue Biol 24: 219-224, 2015

13. Shikinami Y, Matsusue Y and Nakamura T: The complete process of bioresorption and bone replacement using devices made of forged composites of raw hydroxyapatite particles/poly 1-lactide (F-u-HA/PLLA). Biomaterials 26: 5542-5551, 2005.

14. Suljovrujić E, Ignjatović N, Uskoković D, Mitrić M, Mitrović M and Tomić S: Radiation-induced degradation of hydroxyapatite/poly L-lactide composite biomaterial. Radiat Phys Chem 76: 722-728, 2007.

15. Hooper KA, Cox JD and Kohn J: Comparison of the effect of ethylene oxide and $\gamma$-irradiation on selected tyrosine-derived polycarbonates and poly(L-lactic acid). J Appl Polym Sci 63: 1499-1510, 1997. 